\title{
ОЦЕНКА ТЕПЛОВЫХ ПОТЕРЬ ТРУБЧАТОГО ПРИЁМНИКА ТЕПЛА СОЛНЕЧНЫХ ПАРАБОЛОЦИЛИНДРИЧЕСКИХ МОДУЛЕЙ
}

\author{
Л.И. Кныш, докт. техн. наук
}

Днепровский национальный университет имени Олеся Гончара, 49000, пр. Гагарина, 72, г.Днепр, Украина.

Составлена одномерная математическая модель, разработан численный алгоритм и проведены численные эксперименты по количественной оченке тепловых потерь от трубчатого теплоприёмника системы приёма тепла солнечного параболоиилиндрического модуля. Исследования проводились для теплоприёмника, который помещён в стеклянный конверт по типу «труба в трубе». Рассмотрены случаи со слоем атмосферного воздуха в межтрубном пространстве и с вакуумом различной степени. Отдельно проведен расчет для одиночной трубы без стеклянного конверта. Система нелинейных алгебраических уравнений, которая составляет основу математической модели, решена методом простой итерации. Сходимость итераџий обеспечивалась заданием начальных приближений, максимально близких к искомым значениям. В модели предусмотрен учет зависимости теплофизических параметров от температуры и давления. Предложен метод расчета теплового потока через слой газа различной степени разреженности. В качестве физической модели для численных расчетов выбрана модель параболочилиндрического модуля, который исследовался экспериментально и описан в научной литературе. Численные расчеты проведены для случая отсутствия теплового потока от концентратора и при наличие конщентрированного теплового потока. Проведенное сравнение численных $и$ экспериментальных данных показало хорошее совпадение результатов. Незначительное расхождения наблюдалось по значению температуры стекла и по значению тепловых потерь при наличии ветра. Проведенные расчеты показали, что наличие стеклянного пакета является обязательным проектным элементом системы приёма параболоцилиндрических энергетических модулей. Использование такого стеклянного конверта с атмосферным воздухом снижает тепловые потери в два раза, а с глубоким вакуумом - в три раза. Библ. 13, рис. 3.

Ключевые слова: параболочилиндрический концентратор, трубчатый теплоприёмник, стеклянный конверт, тепловые потери, одномерная математическая модель, система нелинейный уравнений, численное решение.

\section{ESTIMATION OF HEAT LOSSES FOR THE TUBE HEAT RECEIVER OF THE SOLAR TROUGH MODULES}

L. Knysh, doctor of technical sciences

Oles Honchar Dnipro National University, 49000, Gagarin ave., 72, Dnipro, Ukraine.

The one-dimensional mathematical model for the quantitative estimation of the heat losses from tube receiver system of the solar trough module was created. Based on this mathematical model the numerical algorithm was developed and numerical experiments were carried out. The studies were conducted for tube heat receiver which placed inside glass envelope as «tube-in-tube». Cases with atmosphere air in annular space and with various vacuum degree were described. Analysis for a unit tube without the glass envelope was carried out separately. A system of nonlinear algebraic equations, which consist basis of the mathematical model, was solved using fixed-point iteration method. Convergence of iterates was provided through initial guess, which were chosen as close to the real values. The thermophysical parameters depend on the temperature and pressure. It was considered in the created mathematical model. The calculation method for the heat flow through a gas layer was offered. The vacuum gas degree was taken into account in the method. As physical model for numerical analysis was chosen solar though module which studied experimentally. Data of the experiments were described in scientific literature. Numerical analyses were carried out for two cases - with heat flow from concentrator and without it. Comparison of numerical and experimental data show good agreement of results. Slight discrepancies were observed for glass temperature and for heat losses in the presence of wind. Analyses show that glass envelope is mandatory design element for heat receiver system of the solar though energy modules. If atmosphere air is used inside glass envelope than heat losses decreased twice, if deeper vacuum - in tree times. Ref. 13, fig. 3.

Keywords: though concentrator, tube heat receiver, glass envelope, heat losses, one-dimension mathematical model, system of nonlinear equations, numerical solution. 


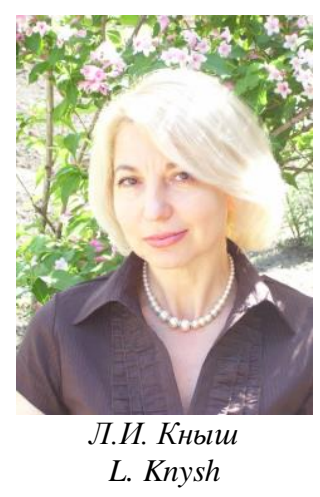

Сведения об авторе: д.т.н., проф., зав. каф. аэрогидромеханики и энергомассопереноса Днепровскогонационального университета имени Олеся Гончара.

Образование: Специалист в области математического и компьютерного моделирования процессов аэрогидродинамики и тепломассообмена.

Научная сфера: исследования процессов переноса в энергетических системах на основе возобновляемых источников энергии.

Публикации: более 100 .

ORCID: 0000-0003-3525-4804

Контакты: тел./факс: +380990034310

e-mail: lknysh@ukr.net
Author information: Doctor of Technical Science, Professor, Chair of Department of Aehydromechanics and Energy Mass Transfer of Oles Gonchar Dnipro National University.

Education: Expert in the field of mathematical and computational modeling of fluid dynamics and heat and mass transfer processes.

Research area: Research energy transfer processes in systems based on the renewable sources energy. Publications: over 100.

ORCID: 0000-0003-3525-4804

Contacts: phone/fax: +380990034310

e-mail: lknysh@ukr.net
Введение. Термодинамическое преобразование энергии Солнца является технологией, которая активно развивается в Украине и за её пределами [1]. Всё чаще плоские и вакуумированные солнечные коллекторы выступают как альтернатива традиционным системам горячего водоснабжения, обеспечивая бесперебойную подачу в автономном режиме $[9,2,7]$. Широкое внедрение получают солнечные термодинамические системы в различных технологических процессах сушке сельскохозяйственной продукции, обогреве теплиц и пр. [8]. Однако подобные энергетические установки с концентраторами ещё не нашли достаточного применения. Существует множество проектов автономных солнечных термодинамических систем с концентраторами разных типов [5]. Но достаточно апробированными являются пока лишь солнечные параболоцилиндрические электрические станции, строительством которых занимаются частные компании Испании, США, Израиля. Основным элементом таких станций является система приёма солнечного излучения «параболоцилиндрический концентратор - трубчатый теплоприёмник» (ПЦК-ТТП). Качество системы приёма во многом определяет эффективность всей энергетической установки. Очевидно, что совершенствование системы приёма, в конечном счёте, приведёт и к более широкому применению параболоцилиндрических энергетических модулей в различных теплообменных системах, расширит их температурных диапазон и энергетические возможности.

Одной из важнейших задач проектирования ПЦК-ТТП является минимизация тепловых потерь от трубчатого теплоприёмника. Решению этой задача посвящена данная работа.

Постановка задачи. В качестве физической модели была рассмотрена классическая система приёма солнечного излучения, состоящая из параболоцилиндрического концентратора, в фокусе которого расположен трубчатый теплоприёмник. Солнечные лучи отражаются от поверхности концентратора и перенаправляются на теплоприёмник, внутри которого движется теплоноситель. Этот теплоноситель может быть рабочим телом одноконтурного или двухконтурного паротурбинного цикла. Кроме того, такая схема может быть использована для других теплоэнергетических целей - подогрев теплоносителя или получения технологического пара, комбинированное термофотоэлектрическое преобразование и пр.

Существует множество подходов к моделированию теплопереноса в системе ПЦК-ТТП [10, 11]. Многие из них слишком детализированные, базируются на стандартных коммерческих вычислительных пакетах, доступ к которым ограничен. В работе [3] предложена математическая модель обобщённого вида, на основе которой создан программный модуль для расчёта основных динамических, геометрических и энергетических показателей системы ПЦК-ТТП. При составлении математической модели не были учтены некоторые моменты, связанные с минимизацией тепловых потерь от трубчатого теплоприёмника. В частности, в [3] для упрощения расчётов принималось, что в фокусе располагается единичная теплоприёмная труба, в то время как такая труба, как правило, располагается в стеклянном конверте по принципу «труба в трубе». Это существенно минимизирует тепловые потери, что повышает общую эффективность установки. Значение тепловых потерь будет ещё более снижено, если систему «труба в трубе» дополнительно вакуумировать. Количественная оценка таких потерь может быть проведена на основе одномерной стационарной модели теплопереноса в трубчатом теплоприёмнике.

Математическая модель теплопереноса в трубчатом теплоприёмнике системы ПЦКТТП. Математическая модель теплообмена между внешней поверхностью канала с теплоносителем и внешней средой было создана на основе принципа суперпозиций. Система уравнений теплового баланса для системы ПЦК-ТТН имеет вид: 


$$
\left\{\begin{array}{l}
Q_{1}+Q_{2}=Q_{3}+Q_{4} \\
Q_{S U N}-Q_{T H}=Q_{3}+Q_{4}
\end{array},\right.
$$

где $Q_{1}, Q_{3}$ - конвективные потери в межтрубном пространстве и между защитным стеклом и окружающей средой соответственно; $Q_{2}, Q_{4}$-лучистые потери в межтрубном пространстве и между защитным стеклом в внешней средой соответственно; $Q_{S U N}-$ тепловой поток от концентратора; $Q_{T H}-$ тепловой поток, поглощенный теплоносителем.

Следует отметить, что в модели не проводился учет теплопроводности защитного стекла.

Радиационные тепловые потери, приходящие на метр теплоприёмника, вычислялись по закону Стефана-Больцмана. Конвективные потери между стеклом и окружающей средой вычислялись с использованием классических критериальных уравнений для случая свободной или вынужденной конвенции.

Величина конвективных потерь в межтрубном пространстве связанна с величиной степени разреженности воздуха в этой зоне. При давлениях, близких к атмосферному, там имеет место свободная конвекция с ламинарным режимом течения. Т.е. основным механизмом теплопереноса в межтрубном пространстве является молекулярная проводимость. Для случая узкого кольцевого канала предлагается использовать подход, описанный в [12]. В нём учет свободноконвективных потоков проводится с использованием коэффициента эффективной теплопроводности вида:

$$
\lambda_{\text {eff }}=0,386 \lambda\left(\frac{P r}{0,861+P r}\right)^{0,25} R a^{* 0,25},
$$

где $\lambda$ - коэффициент теплопроводности среды, в данном случае воздуха; $P r$ - число Прандтля среды; $R a^{*}$ - число Рэлея специального вида:

$$
R a^{*}=\frac{\left(\ln \left(\frac{D_{C T}}{D_{T P}}\right)\right)^{4}}{L^{3}\left(D_{T P}^{-0,6}+D_{C T}^{-0,6}\right)^{5}} R a_{T P} .
$$

В последнем выражении $D_{T P}, D_{C T}$ - диаметры единичной трубы и системы «труба в трубе» соответственно; $L_{-}$длина исследуемого участка трубы; $R a_{T P}$ - число Рэлея при температуре стенки единичной трубы.

Окончательно выражения для определения конвективных потерь в межтрубном простран- стве по форме соответствует выражению для вычисления молекулярной теплопрооводности:

$$
Q_{1}=\frac{2 \pi \lambda_{e f f}}{\ln \left(\frac{D_{C T}}{D_{T P}}\right)}\left(T_{T P}-T_{C T}\right),
$$

где $T_{T P}$ и $T_{C T}$ - температуры трубы с теплоносителей и стеклянной трубы соответственно.

Следует отметить, что для расчёта эффективного коэффициента теплопроводности может быть использована также классическая эмпирическая формула М.А. Михеева [6]. Однако проведенные численные исследования показали, что предложенная методика более точно соответствует эксперименту, так как учитывает цилиндрическую геометрию системы.

Уменьшение давления в межтрубном пространстве приводит к изменению механизма теплопередачи. Постепенно длина свободного пробега молекул начинает увеличиваться. При этом свободная конвекция ослабевает - фактически не хватает молекул для транспортировки энергии. Начинает доминировать свободно-молекулярный режим, при котором межмолекулярные взаимодействия почти не происходят. Считается, что молекулы просто двигаются между двумя поверхностями. Расчёт теплового потока для такого режима проводиться, как правило, по упрощённым формулам из кинетической теории газов вида [4]:

$$
q=\left(c_{v}+\frac{1}{2} R\right) \bar{\rho} \sqrt{\frac{R T}{2 \pi}} a\left(T_{T P}-T_{C T}\right),
$$

где $c_{v}$ - изохорная теплоёмкость; $\bar{\rho}-$ средняя плотность газа; $a$ - коэффициент аккомодации; $T$ - температура газа вблизи внутренней поверхности трубы; $R$ - газовая постоянная.

Однако для данной задачи использование таких соотношений затрудненно. Поэтому предлагается подход, основанный на использовании коэффициента теплопередачи через слой разреженного газа:

$$
K=\frac{\lambda}{\frac{D_{T P}}{2} \ln \frac{D_{C T}}{D_{T P}}+b l\left(\frac{D_{T P}}{D_{C T}}+1\right)},
$$

где $l$ - длина свободного пробега молекул, а $b$ - безразмерный коэффициент, который определяется выражением:

$$
b=\frac{2-a}{a} \cdot \frac{9 \gamma-5}{2(\gamma-1)}
$$

где $\gamma$ - показатель адиабаты. 
Нахождение значения коэффициента аккомодации, особенно для селективных поверхностей, является отдельной задачей. Но следует отметить, что этот коэффициент относительно слабо характеризует реальный тепломассоперенос в межтрубном пространстве. Многие литературные источники и экспериментальные данные указывают на то, что для хорошо очищенных поверхностей значение коэффициента аккомодации близко к единице. Именно такое предположение было принято в данной работе.

В качестве критерия, характеризующего переход от конвективного теплообмена к свободномолекулярному в межтрубном пространстве, использовалось число Рэлея $R a$. Для $R a \geq 100$ использовалась конвективная модель, а для $R a<100$ - кинетическая теория. Конечно, между этими режимами теплообмена существует некоторая переходная зона. Её наличие учитывалась косвенно в выражении для коэффициента теплопередачи $K$.

Выбор численного метода и его сходимость. На основе приведенной выше математической модели был создан расчётный алгоритм, состоящий из трёх основных блоков. В первом блоке помещались значения теплофизических констант, зависящих от температуры; во втором блоке - критериальные уравнения, вид которых определялся природой теплообмена; в третьем блоке непосредственно задавались начальные данные и проводился расчёт. Нелинейная система балансовых уравнений решалась методом простой итерация. Теоретически вопрос сходимости этого метода не исследовался. Сходимость достигалась максимальным приближением начальных значений к физически реальным.

Анализ результатов численного моделирования. Численное исследования на основе созданной математической модели проводились с использованием экспериментального параболоцилиндрического модуля, который тестировался в Sandia National Laboratories (США) [13]. Длина параболического модуля составляла 7,8 м, диаметр апертуры 5 м. Рассматривался металлокерамический трубчатый теплоприёмник, расположенный на фокусной линии модели. На внешней стороны теплоприёмника было нанесено градиентное селективное покрытие. Такие теплоприёмники имеют более высокие эксплуатационного характеристики, чем традиционное черные хромовые. Кроме того, селективное покрытие способно минимизировать радиационные потери, являеться актуальным в такие системах. Единичная металлокерамическая труба диаметром 0,07 м располагалась внутри стеклянной трубы. Внешний диаметр системы «труба в трубе» равнялся 0,109 м. Предполагалось, что в межтрубном пространстве может находится воздух при различных давлениях. В качестве теплоносителя использовалось синтетическое масло Syltherm 800.

Для удобства сравнения и верификации созданной математической модели и численного алгоритма был выбран уровень температур, который соответствовал экспериментальным исследованиям, описанным в [13]. Температура окружающей среды равнялась $22{ }^{\circ} \mathrm{C}$, а так называемая sky-температура была $14{ }^{\circ} \mathrm{C}$. При расчётах учитывалась зависимость степени черноты трубы с теплоносителем от температуры.

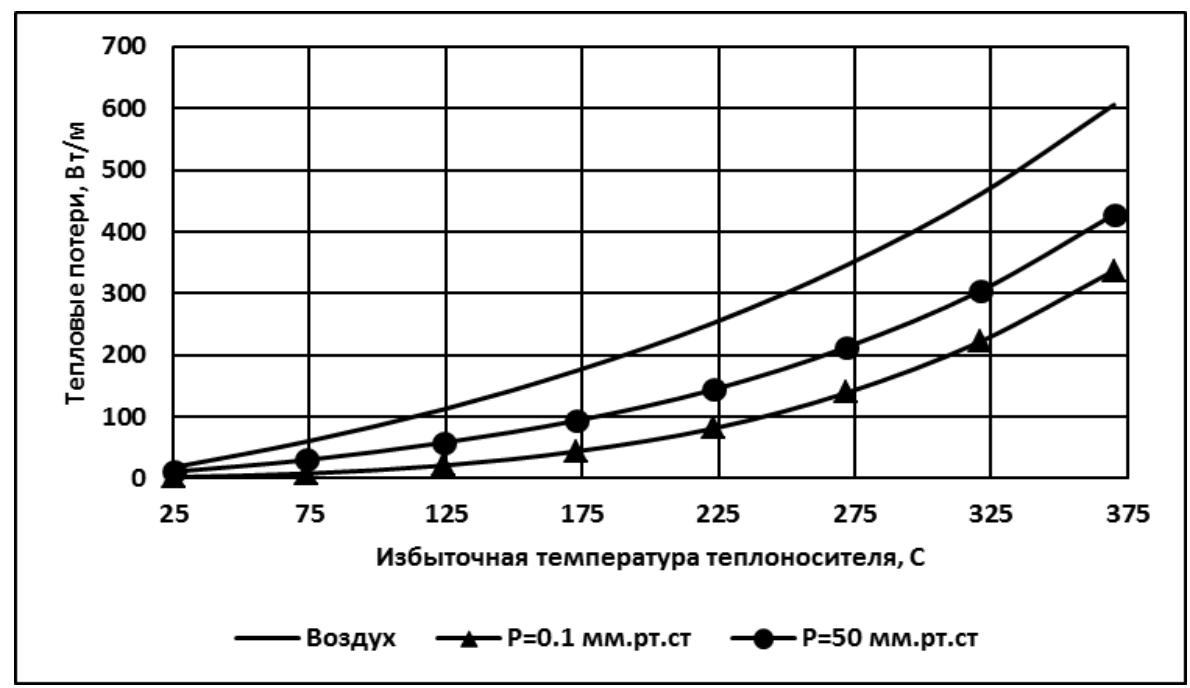

Рис. 1. Зависимость тепловых потерь от избыточной температуры при различном уровне вакуума при отсутствии теплового потока от концентратора.

Fug. 1. Thermal losses Vs. heat receiver temperature above ambient for various vacuum degree without the heat flow from concentrator. 
На рис. 1 представлены результаты первого цикла расчётов тепловых потерь трубчатого теплоприёника. Численные эксперименты в этом случае проводились при отсутствии теплового потока от концентратора, в безветренную погоду - учитывалась лишь свободная конвекции вокруг теплоприёмника. Предполагалось, что теплоноситель не циркулирует в канале. В этом случае второе уравнение в общей системе уравнений баланса отсутствует, что позволяет детально проанализировать влияние степени разряжения в межтрубном пространстве на общие тепловые потери в теплоприёмнике.

В расчётах уровень значения давления изменялся в широком диапазоне. На рис.1 представлены три характерные кривые - воздух в межтрубном пространстве при нормальном атмосферном давлении, при давлении, характерном для переходного режима и при давлении, которое соответствует глубокому вакууму. Можно заметить, что тепловые потери при создании глубокого вакуума снижаются почти в два раза, что, очевидно, существенно повышает общую эффективность системы.

Расчётные значения тепловых потерь, полученные для воздуха и вакуума, практически совпадают с экспериментальными результатами, полученными в [13]. Модель имеет некоторую погрешность лишь при вычислении температуры стеклянной трубы. На рис. 2 представлено отклонение между расчётными и экспериментальными данными.

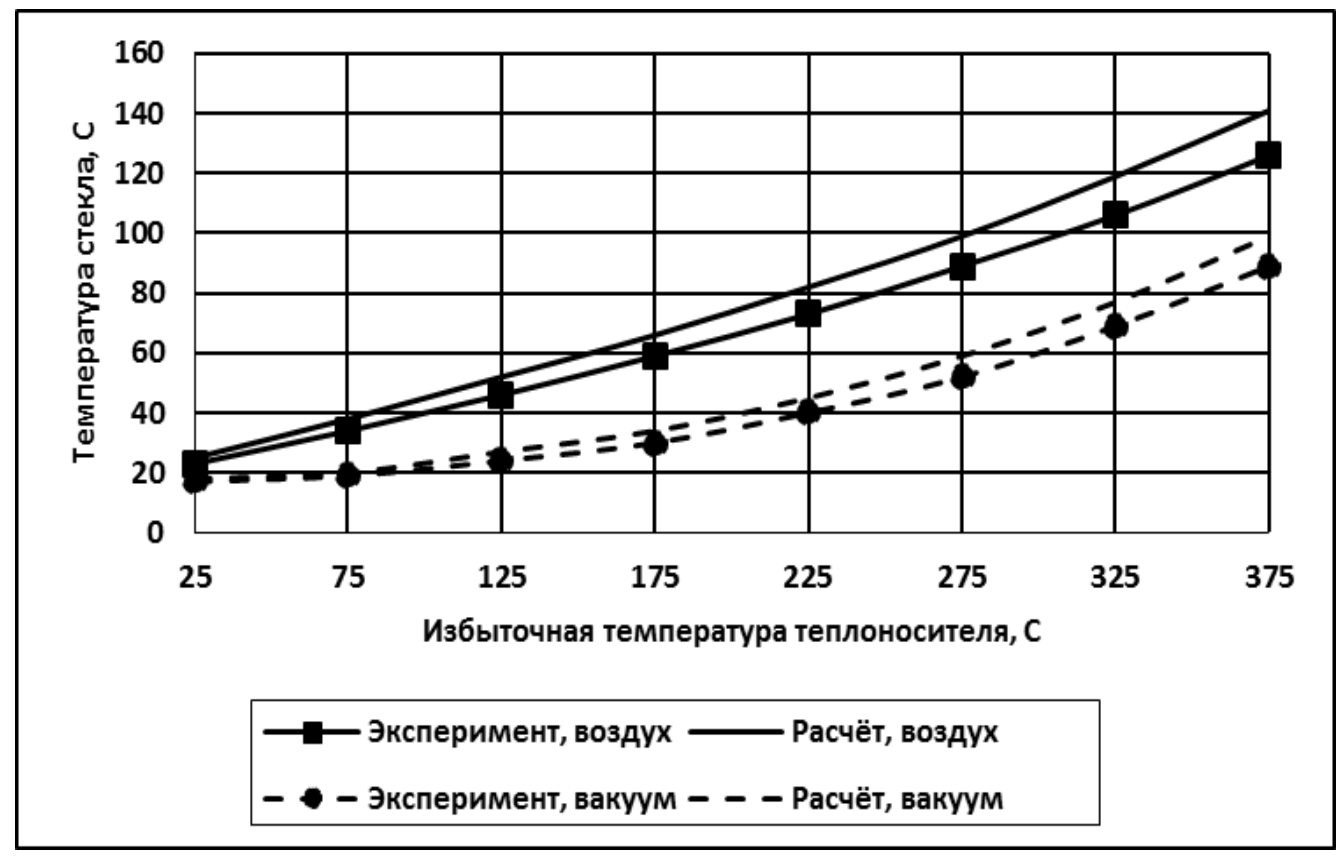

Рис. 2. Сравнение экспериментальной и расчётной абсолютной температуры поверхности стекла.

Fug. 2. Comparison experimental and calculated temperature of the glass surface.

Как видно из графика, расчётные данные являются несколько завышенными, как для случая использования воздуха при нормальном атмосферном давлении, так и для вакуумированного межтрубного пространства. Расхождения наблюдаются главным образом при повышенных температурах, где отклонения приближаются к $10 \%$. Очевидно, такие отклонения связаны с использованием одномерной модели. Использование более детализированной методика, например, предложенной в [3], приблизит значения температур к экспериментальным.

Аналогичные расчёты были проведены и для случая, когда тепловой поток от концен- тратора поступал на теплоприёмный канал. Предполагалось, что плотность теплового потока от концентратора равнялась $940 \mathrm{BT} / \mathrm{m}^{2}$. Такое значение выбиралось для удобства сравнения предлагаемой методики и экспериментальных данных, представленных в [13]. На рис. 3 представлены результаты расчёта тепловых потерь системы ПЦК-ТТП для экспериментальной установки. Рассматривался случай отсутствия ветра. В этом случае данные расчёта практически полностью совпадали в экспериментальными данными. При ветреной погоде наблюдалось некоторое расхождение данных, которое находилось в пределах 10-15\%. 


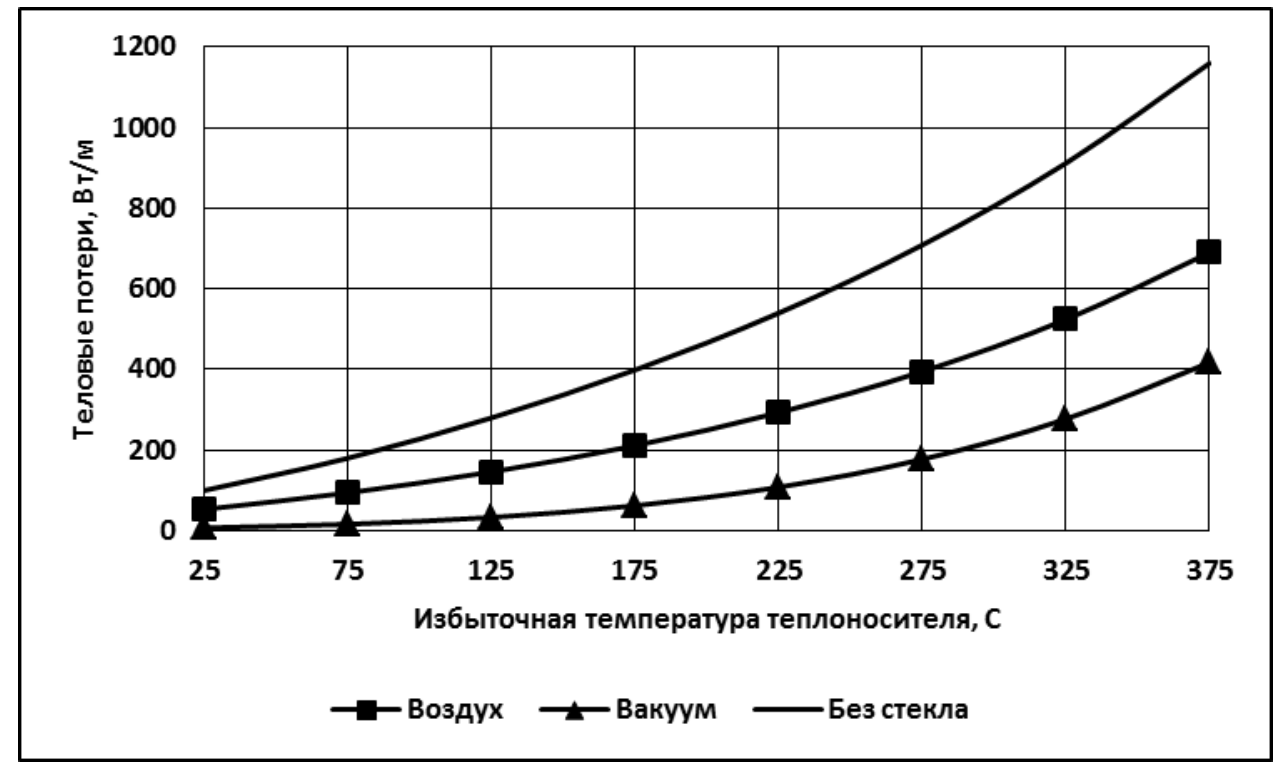

Рис. 3. Зависимость тепловых потерь от избыточной температуры теплоносителя при наличие теплового потока от концентратора.

Fug. 3. Thermal losses Vs. heat receiver temperature above ambient with heat flow from concentrator.

На рис. 3 также представлены данные для случая, когда защитное стекло отсутствует. Как видно, в этом случае тепловые потери увеличиваются практически в два раза по сравнению со случаем воздушной прослойки в межтрубном пространстве и в три раза для вакуумированного зазора.

Очевидно, что несмотря на технологическое усложнение системы, вакуумирование дает существенный прирост эффективности за счёт снижения тепловых потерь. Но следует учесть, что вакуумирование эффективно лишь при отсутствии притока водорода из окружающего воздуха в зазор. Термические потери с водородом в межтрубном пространстве могут быть даже выше кондуктивных потерь с воздухом.

Выводы. Проведенное исследование является логическим продолжение работ автора по комплексному исследованию тепломассопереносу в системах приёма солнечных термодинамических модулей с параболоцилиндрическими концентраторами. Разработанная методика теоретически обосновывает необходимость использования стеклянного конверта для канала с теплоносителем при проектировании подобных энергетических систем. Элементы представленной математической модели могут быть использованы в качестве граничных условий для более детализированных расчётов. Созданный численный алгоритм, который базируется на решении системы нелинейных алгебраических уравнений, может быть легко адаптирован для расчёта систем ПЦК
- ТТП произвольной геометрии, различной мощности и назначения.

1. Будько В.И., Кудря С.А., Пепелов А.В. Современное состояние и развитие возобновляемой энергетики. Альтернативная энергетика и экология. 2017. № 4-6. C. $130-141$.

2. Кныли Л.И. Метод учёта тепловой проводимости абсорбера в плоском солнечном коллекторе. Відновлювальна енергетика. 2014. № 2(37). С. 38-42.

3. Кныш Л.И., Гоман О.Г. Особенности моделирования энергопереноса в системе приёма тепла солнечной параболоцилиндрической станции. Вісник Херсонського національного технічного університету. 2016. № 3(58). C. 352-356.

4. Кошмаров Ю.А., Рыжсо Ю.А. Прикладная динамика разреженного газа. М. Машиностроение. 1977. 184 с.

5. Кудря С.А., Пепелов А.В. Використання відновлюваної енергії: досвід розвинутих країн. Київ. ЮНІДО. 2015. 32 p. URL: http://www.reee.org.ua/download/trainings/ $\% \mathrm{D} 0 \% \mathrm{~A} 2 \% \mathrm{D} 0 \% 9 \mathrm{C} 28 . \mathrm{pdf}$

6. Михеев М.А., Михеева И.М. Основы теплопередачи. М. Энергия. 1977. 344 с.

7. Рєзиов В.Ф., Суржик Т.В., Охота О.О. Експериментальне дослідження теплопровідності композіційних матеріалів колекторів сонячної енергії. Відновлювальна енергетика. 2016. № 2(46). С. 41-44.

8. Рєзиов В.Ф., Суржик Т.В., Щокіна В.А. Можливі причини формування неоднорідних структур при геліосушці вологовмісних середовищ. Відновлювальна енергетика. 2015. № 1(40). С.28-31.

9. Суржик Т.В. Дослідження матеріалу абсорбера сонячного колектора 3 полімерних композиційних матеріалів. Відновлювальна енергетика. 2015. № 4(44). С.28-31.

10. Hachicha A.A., Bashria A.A. Yousef, Zafar Said, Ivette Rodríguez. A review study on the modeling of high-temperature solar thermal collector systems. Renewable and Sustainable Energy Reviews. 2019. V. 112. Pp. 280-298. 
11. Cheng Z.D., He Y.L., Xiao J., Tao Y.B., Xu R.J. Threedimensional numerical study of heat transfer characteristics in the receiver tube of parabolic trough solar collector. Int. J. Heat Mass Transf. 2010. V. 37. Pp. 782-787.

12. Theodore L. Bergman and other. Fundamentals of Heat and Mass Transfer. John Wiley \& Sons. 2007. 1051 p.

13. Dudley V., Kolb G., Sloan M., Kearney D. SEGS LS2 Solar Collector Test Results. Report of Sandia National Laboratories. USA. 1994. 140 c.

\section{REFERENCES}

1. Budko V.Y., Kudria S.A., Pepelov A.V. Sovremennoe sostoyanie i razvitie vozobnovlyaemoj energetiki. [Current state and development of renewable energy]. Alternativnaya energetika i ekologiya. 2017. No. 4-6. Pp. 130-141. [in Russian].

2. Knysh L.I. Metod uchyota teplovoj provodimosti absorbera $\mathrm{v}$ ploskom solnechnom kollektore. [The method of accounting of heat conductivity of absorber the flat solar collector]. Vidnovluvana energetika. 2014. No. 2(37). Pp. 38-42. [in Russian].

3. Knysh L.I., Goman O.G. Osobennosti modelirovaniya energoperenosa $\mathrm{v}$ sisteme priyoma tepla solnechnoj paraboloczilindricheskoj stanczii. [Modeling features of energy-mass transfer in the heat receiver system of the solar parabolic cylindrical plant]. Visnyk Khersonskoho natsionalnoho tekhnichnoho universytetu. 2016. No. 3(58). Pp. 352-356. [in Russian].

4. Koshmarov Yu.A., Ryzhov Yu.A. Prikladnaya dinamika razrezhennogo gaza. [Applied rarefied gas dynamics]. Moscow. Mashinostroenie. 1977. 184 p. [in Russian].

5. Kudria S.A., Pepelov A.V. Vykorystannia vidnovliuvanoi enerhii: dosvid rozvynutykh krain. [The use of renewable energy: the experience of developed countries]. Kiev. UNIDO. URL: http://www.reee.org.ua/download/trainings/\%0\% A2\%D0\% 9C28.pdf . [in Ukrainian]

6. Mikheev M.A., Mikheeva I.M. Osnovy teploperedachi. [Heat Transfer Basics]. Moscow. Energiya. 1977. 344 p. [in Russian].

7. Rieztsov V.F., Surzhyk T.V., Okhota O.O. Eksperymentalne doslidzhennia teploprovidnosti kompozitsiinykh materialiv kolektoriv soniachnoi enerhii. [Experimental study of thermal conductivity of composite materials of solar energy collectors]. Vidnovluvana energetika. 2016. No. 2(46). Pp. 41-44. [in Ukrainian].

8. Rieztsov V.F., Surzhyk T.V., Shchokina V.A. Mozhlyvi prychyny formuvannia neodnoridnykh struktur pry heliosushtsi volohovmisnykh seredovyshch. [Possible reasons for the formation of inhomogeneous structures during the solar drying of moisture-containing media]. Vidnovluvana energetika. 2015. No. 1(40). Pp. 28-31. [in Ukrainian].

9. Surzhyk T.V. Doslidzhennia materialu absorbera soniachnoho kolektora $\mathrm{z}$ polimernykh kompozytsiinykh materialiv. [Investigation of the material of a solar collector absorber made of polymer composite materials]. Vidnovluvana energetika. 2015. No. 4. Pp. 28-31. [in Ukrainian].

10. Hachicha A.A., Bashria A.A. Yousef Zafar Said, Ivette Rodriguez. A review study on the modeling of high-temperature solar thermal collector systems. Renewable and Sustainable Energy Reviews. 2019. V. 112. Pp. 280-298. [in English].

11. Cheng Z.D., He Y.L., Xiao J., Tao Y.B., Хu R.J. Three-dimensional numerical study of heat transfer characteristics in the receiver tube of parabolic trough solar collector. International Journal of Heat and Mass Transfer. 2010. V. 37. Pp. 782-787. [in English].

12. Theodore L., Bergman and other. Fundamentals of Heat and Mass Transfer. John Wiley \& Sons. 2007. 1051 p. [in English].

13. Dudley V., Kolb G., Sloan M., Kearney D. SEGS LS2 Solar Collector Test Results. Report of Sandia National Laboratories. USA. 1994. 140 p. [in English].

\section{ОЦНКА ТЕПЛОВИХ ВТРАТ ТРУБЧАТОГО ПРИЙМАЧА ТЕПЛА СОНЯЧНИХ ПАРАБОЛОЦИЛІНДРИЧНИХ МОДУЛІВ}

\section{Л.І. Книш, докт. техн. наук}

Дніпровський національний університет імені О. Гончара, 49000, пр. Гагаріна, 72, м. Дніпро, Украина

Складено одновимірну математичну модель, розроблено числовий алгоритм та проведено числові експерименти по визначенню кількісних показників теплових втрат від трубчатого теплоприймача системи прийому тепла сонячного параболоииліндричного модуля. Дослідження проводились для теплоприймача, який знаходиться в середині скляного конверту по типу «труба в трубі». Розглянуто випадки зі шаром атмосферного повітря в міжтрубному просторі та із вакуумом різного ступеню. Окремо проведено розрахунок для одиничної труби без скляного конверту. Система нелінійних алгебраїчних рівнянь, яка складає основу математичної моделі, розв'язана методом простої ітераиії. Збіжність ітерачій забезпечувалась заданням початкових наближень, максимально наближених до шуканих значень. В моделі було передбачено врахування залежності теплофізичних параметрів від температури та тиску. Запропоновано метод розрахунку теплового потоку скрізь шар газу різного ступеня розрідженості. В якості фізичної моделі для числових розрахунків вибрано модель параболоииліндричного модуля, який був досліджений експериментально та описаний в науковій літературі. Числові розрахунки проведено для випадку відсутності теплового потоку від концентратору та при наявності кониентрованого теплового потоку. Проведено порівняння числових та експериментальних даних, яке показало добрий збіг результатів. Незначна розбіжність спостерігалась по значенню температури скла та по значенню теплових втрат при наявності вітру. Розрахунку, які були проведені, показали, що наявність скляного пакету $\epsilon$ обов'язковим проектним елементом системи прийому параболочиліндричних енергетичних модулів. Використання такого скляного конверту з атмосферним повітрям знижує теплові втрати в два рази, а з глибоким вакуумом - в три рази. Бібл. 13, рис. 3.

Ключові слова: параболочиліндричних концентратор, трубчатий теплоприймач, скляний конверт, теплові втрати, одновимірна математична модель, система нелінійних рівнянь, числовий розв'язок. 